\title{
Reassessment of biochemically determined Hunter syndrome carrier status by DNA testing
}

\author{
Kirsten M Timms, Faye J Edwards, John W Belmont, John R W Yates, Richard A Gibbs
}

\begin{abstract}
Deficiency of iduronate-2-sulphatase (IDS) results in the $X$ linked recessive lysosomal storage disorder Hunter syndrome. Determination of carrier status in families affected by this disorder has been performed using a variety of enzymatic tests. None of these tests has proved to be $100 \%$ effective at identifying carriers. The aim of this study was to perform carrier testing in a family affected by the disorder, where testing was complicated by the fact that no surviving affected subjects were available for study. Direct dye primer sequencing of $P C R$ products was used to identify mixed bases in an obligate carrier. Two mixed bases were observed within exon VIII. The first base change $(T \rightarrow A)$ at nucleotide position 1150 results in a missense mutation (H342Q), while the second base change $(G \rightarrow T)$ at nucleotide position 1151 results in a nonsense mutation (G343X). Four additional female family members were screened for the same mutation. Using this approach it is possible to provide unambiguous information about a subject's carrier status and, unlike biochemical testing, this approach will be equally effective when applied to families with the mild form of this disorder.
\end{abstract}

$(\mp$ Med Genet 1998;35:646-649)

Keywords: Hunter syndrome; carrier analysis; iduronate-2-sulphatase

Department of Molecular and Human Genetics, S930, Baylor College of Medicine, One Baylor Plaza, Houston, TX 77030, USA

K M Timms

F J Edwards

J W Belmont

R A Gibbs

Department of Pathology, University of Cambridge, Box 134, Cambridge CB2 2QQ, UK

J R W Yates

Correspondence to: Dr Timms, Myriad Genetics Inc, 390 Wakara Way, Salt Lake City, UT 84108, USA.

Received 18 November 1997 Revised version accepted for publication 13 January 1998 using a number of biochemical tests. These include analysis of IDS activity in serum ${ }^{6}$ or hair roots, ${ }^{78}$ cloning analysis of cultured fibroblasts, ${ }^{911}$ and a method based on inhibition of cross correction of the enzyme defect between the two cell populations present in cultured fibroblasts from carriers. ${ }^{12}{ }^{13}$ To date, none of the available enzymatic tests has been shown to identify accurately $100 \%$ of obligate carriers. In addition, testing of cultured fibroblasts has been shown to diagnose some

A

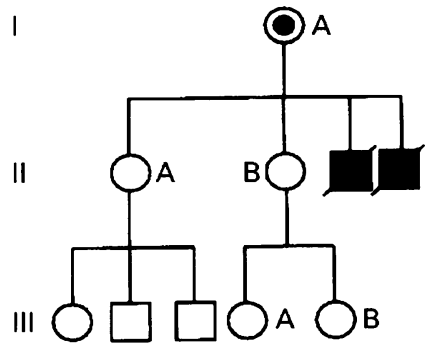

B

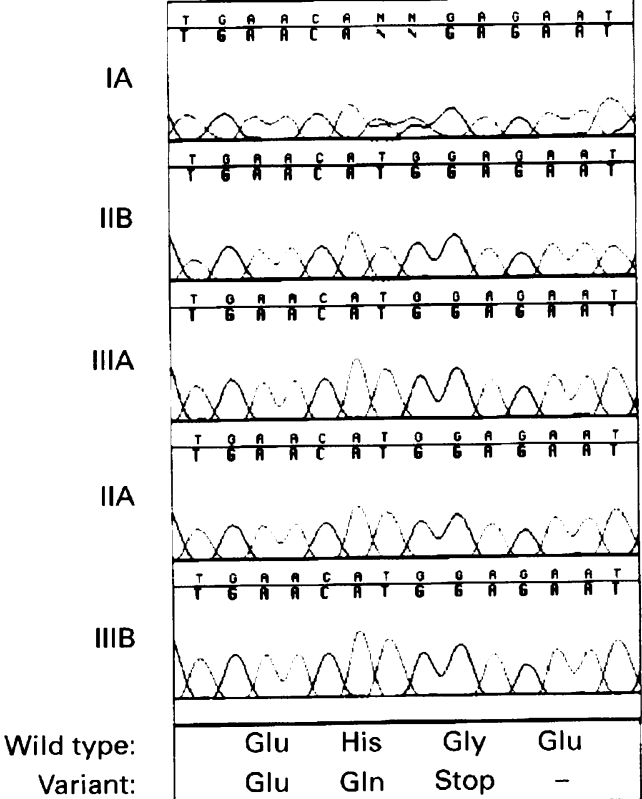

Figure 1 (A) Hunter syndrome pedigree. $L A$ is an obligate carrier female; filled squares are affected males. Mutation analysis was performed on DNA from $I A$, the obligate carrier. Subjects IIA, IIB, IIIA, and IIIB were analysed to determine their carrier status. Enzymatic testing had previously been carried out on cultured fibroblasts from IIA IIB, and IIIA. These results indicated that both IIA and IILA were non-carriers, while IIB tested positive as a carrier. (B) Results of sequence analysis on exon $8 P C R$ product from five members of the Hunter syndrome pedigree. Mixed bases in the obligate carrier are indicated by arrows; none of the other female members of the pedigree showed mixed bases at the female members of the pedigree showed mixed bases at the
same positions. The amino acid changes that occur as a result same positions. The amino acid changes that occur as a resu
of the two mutations identified in the obligate carrier are shown below the sequence electrophoretograms. 
Table 1 Oligonucleotides used in this study

\begin{tabular}{lll}
\hline Primer & Sequence & Description \\
\hline R3310 & TGTAAAACGACGGCCAGTCTGCTAACTGCGCCACCTG & Exon 1 primer with universal tail \\
R3311 & CAGGAAACAGCTATGACCGGAAGGGAGAAGAGATGGC & Exon 1 primer with reverse tail \\
R3312 & TGTAAAACGACGGCCAGTCTTAGTAACCTAGCACCTAC & Exon 2 primer with universal tail \\
R3313 & CAGGAAACAGCTATGACCCTGACAATAGCTGAAGCTC & Exon 2 primer with reverse tail \\
R3027 & GCTGTTTGCTAGGAGCCTCG & Exon 3 primer, IDS specific \\
R3235 & GTAATGAGAACACCAAACTG & Exon 3 primer, IDS specific \\
R3314 & TGTAAAACGACGGCCAGTGCTGTTTGCTAGGAGCCTCG & Exon 3 primer with universal tail \\
R3315 & CAGGAAACAGCTATGACCCACTTTGGGTGAAAACGTGGC & Exon 3 primer with reverse tail \\
R3087 & TGTAAAACGACGGCCAGTGGTTCCACTTGCCCATTTG & Exon 4 primer with universal tail \\
R3309 & CAGGAAACAGCTATGACCCAGCTTCACAGAACATGCAG & Exon 4 primer with reverse tail \\
R3316 & TGTAAAACGACGGCCAGTCTGTCTTAGTTCTACTTCTG & Exon 5 primer with universal tail \\
R3317 & CAGGAAACAGCTATGACCCATCAGGCAACCATGGTGC & Exon 5 primer with reverse tail \\
R3318 & TGTAAAACGACGGCCAGTGATAGAGCCACAAGCTTGTG & Exon 6 primer with universal tail \\
R3319 & CAGGAAACAGCTATGACCGTGGAGTTGTGTCTACTGAG & Exon 6 primer with reverse tail \\
R3320 & TGTAAAACGACGGCCAGTCTGAGAAAATCATTAAGGGC & Exon 7 primer with universal tail \\
R3321 & CAGGAAACAGCTATGACCTTCACAGGAAAGTTCAGATG & Exon 7 primer with reverse tail \\
R3322 & TGTAAAACGACGGCCAGTTCTGTGGTAATTCCAAGTG & Exon 8 primer with universal tail \\
R3323 & CAGGAAACAGCTATGACCCCCCCAAAGCCTATGATTC & Exon 8 primer with reverse tail \\
R3324 & TGTAAAACGACGGCCAGTATGTAACCCATTCTGCTCTG & Exon 9 primer with universal tail \\
R3325 & CAGGAAACAGCTATGACCGCTGGAAGGGAGCACATC & Exon 9 primer with reverse tail \\
R3033 & TCTGTGGTAATTCCAAGTG & Exon 8 primer \\
R3034 & CCCCCAAAGCCTATGATTC & Exon 8 primer \\
R4210 & CTGTATTTGGCCCATTCTCAT & Exon 8 variant specific primer \\
R4211 & GTGGGCTCTAGGTGAACAAT & Exon 8 variant specific primer \\
R4213 & CTGTATTTGGCCCATTCTCCA & Exon 8 wild type specific primer \\
R4214 & GTGGGCTCTAGGTGAACATG & Exon 8 wild type specific primer \\
\hline
\end{tabular}

non-carriers of the disease erroneously as carriers. ${ }^{13}$ DNA testing provides an opportunity to identify carriers unambiguously. To perform carrier testing in affected families it is generally necessary to identify the mutant allele. This study used high fidelity dye primer direct sequencing of PCR amplified IDS exons from an obligate carrier to determine the causative mutation. This approach was necessary as no surviving affected subjects were available for study. Following identification of the mutation, carrier analysis was performed on an additional four female family members. Skin biopsies from three of these had previously been analysed, and one subject had tested positive as a carrier using this approach.

\section{Materials and methods \\ SUBJECTS}

Carrier analysis was performed on DNA from five subjects from an extensive pedigree which contains at least nine people affected with severe Hunter syndrome over five generations. The obligate carrier (IA, fig 1) had two children who died of Hunter syndrome at the ages of $31 / 2$ and 11 years. Biochemical testing of skin biopsies had previously been performed on three of the subjects (IIA, IIB, and IIIA). IIB had tested positive as a carrier in these tests. No surviving affected subjects were available for study.

\section{SOUTHERN HYBRIDISATION}

Southern hybridisations were performed using standard techniques. ${ }^{14}$ The IDS cDNA probe was generated as previously described. ${ }^{15}$

\section{PCR}

PCR was carried out in $100 \mu \mathrm{l}$ reactions containing $25 \mathrm{mmol} / \mathrm{l}$ trycine, $\mathrm{pH} 8.7,85$ $\mathrm{mmol} / 1 \mathrm{KOAc}, 1.1 \mathrm{mmol} / \mathrm{l} \mathrm{Mg}(\mathrm{OAc})_{2}, 8 \%$ glycerol, $2 \%$ DMSO, $0.2 \mathrm{mmol} / 1$ each dNTP, $40 \mathrm{pmol}$ each primer, 3.2 U rTth DNA polymerase, and $0.1 \mathrm{U}$ Vent DNA polymerase. AmpliWax® PCR Gem 100s (PE Applied Biosystems, Foster City, CA) were used to facilitate hot start PCR. Approximately $100 \mathrm{ng}$ genomic DNA was used as template. PCR conditions were $95^{\circ} \mathrm{C}$ for three minutes, followed by 35 reaction cycles of $95^{\circ} \mathrm{C}$ for 30 seconds, $55^{\circ} \mathrm{C}$ for 30 seconds, and $68^{\circ} \mathrm{C}$ for 90 seconds. After completion of the cycles the samples were held at $68^{\circ} \mathrm{C}$ for an additional seven minutes and then cooled to $4^{\circ} \mathrm{C}$. The primers used in this study are described in table 1. PCR products were cloned into the vector pT-Adv using the AdvanTAgeTM PCR Cloning Kit (Clontech, Palo Alto, CA) according to the manufacturer's instructions.

\section{DNA SEQUENCING}

PCR products were gel purified using a QIAquick Gel Extraction Kit (Qiagen Inc, Santa Clarita, CA) and then sequenced directly using universal and reverse BODIPY dye primers as previously described. ${ }^{16}$ Dye terminator universal and reverse reads were performed on cloned DNA using reagent kits provided by PE Applied Biosystems (Foster City, CA). Reactions were performed on PEC 9600 thermocyclers and electrophoresed on ABI 373 or 377 DNA sequencers. Sequence analysis was carried out using Sequencher Version 3.0 (Gene Codes Corporation).

\section{Results}

Approximately $20 \%$ of cases of Hunter syndrome are the result of major structural rearrangements (deletions or rearrangements) within the IDS gene. ${ }^{4}$ To determine whether this was the case in this family (fig 1A), Southern hybridisation was carried out on HindIII digested genomic DNA using the complete IDS cDNA as a probe. All five subjects showed hybridisation patterns identical to the control genomic DNA sample (data not shown).

PCR was carried out on genomic DNA from the obligate carrier (IA) with primers spanning each of the nine IDS exons. One primer from each set contained a 5 ' terminal sequence identical to the M13 universal sequencing primer, while the opposite PCR primer contained a 


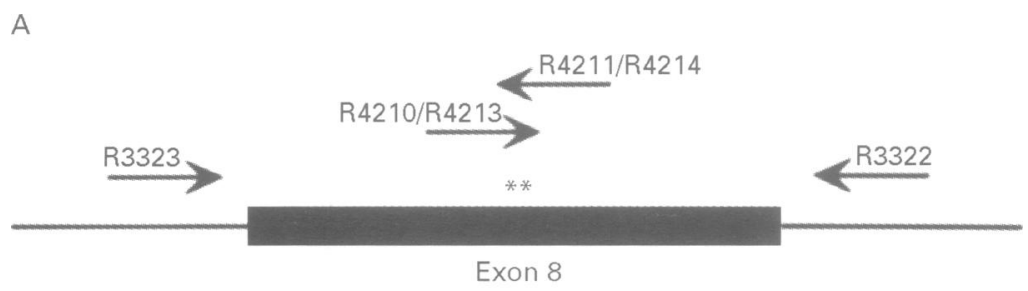

B

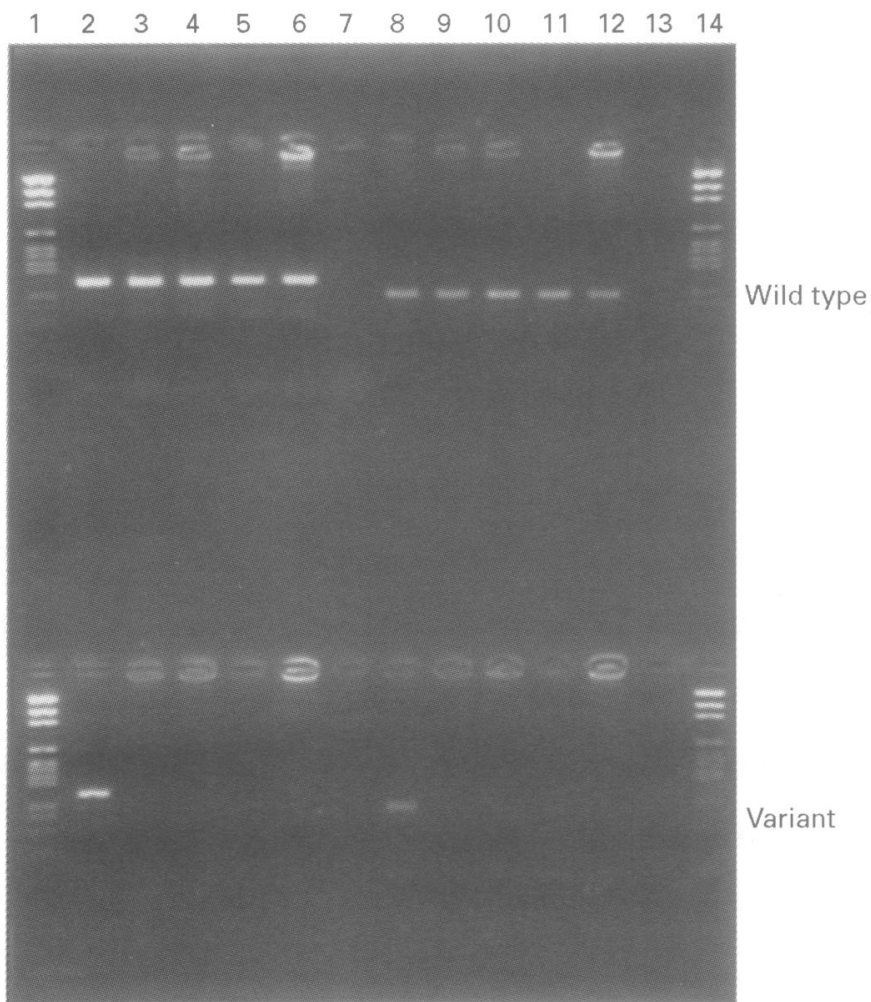

Figure 2 (A) Schematic diagram showing the PCR experiment used to amplify specifically either the normal or mutant allele. Exon 8 is shown as a thick black line, with the flanking introns shown as thin lines. Arrows indicate the position of primers used in the $P C R$ reactions. The two flanking primers (R3322 and $R 3323)$ will amplify from either allele. The internal primers will amplify from either the wild type (R4213 and $R 4214)$ or variant (R4210 and R4211) allele. The location of the mixed bases at the 3 ' ends of the internal primers is indicated by *. (B) Results from the PCR carried out using primer pairs specific for either the wild type or variant allele. Lanes 1 and 14, markers $(2645,1605$, $1198,676,517,460,396,350,222,179$ and $126 \mathrm{bp}$ ); lanes 2 and 8, IA; lanes 3 and 9, IIA; lanes 4 and 10, IIB; lanes 5 and 11, IIIA; lanes 6 and 12, IIIB; lanes 7 and 13, no template. The top half of the gel shows products after amplification with primers which specifically amplify the wild type allele. Lanes $2-7, R 3323+R 4214$; lanes 8-13, R3322 + $R 4213$. The bottom half of the gel shows products after amplification with primers specific for the variant allele. Lanes $2-7, R 3323+R 4211$; lanes $8-13, R 3322+R 4210$.

region identical to the $M 13$ reverse sequencing primer. The presence of the IDS pseudogene located distal to the active gene complicated the analysis in the case of exon 3. This exon was first amplified using primers which are specific for IDS exon 3 and do not amplify the nearly identical region of the IDS pseudogene. A second round PCR was then performed using tailed exon 3 primers and purified first round PCR product as template. Amplification was carried out using high fidelity conditions including the use of a proof reading enzyme to minimise the possibility of PCR error. The resulting PCR products were purified by preparative agarose gel eletrophoresis and then sequenced directly using dye primer chemistry and BODIPY universal and reverse dye primers. ${ }^{16}$ The resulting sequences were compared to the published genomic sequence, ${ }^{3}$ and two mixed bases were observed within exon VIII (fig 1B). The first base change $(T \rightarrow A)$ at nucleotide position 1150 results in a missense mutation with a histidine amino acid being replaced by a glutamine $(\mathrm{H} 342 \mathrm{Q})$. The second base change $(\mathrm{G} \rightarrow \mathrm{T})$ at nucleotide position 1151 results in a nonsense mutation (G343X).

To confirm that these were true mixed bases this exon was reamplified using primers without universal or reverse tails and then cloned into a vector containing universal and reverse sequencing primer sites. Six individual clones were selected and sequenced in both orientations using dye terminator chemistry. Sequence from three of the six clones was identical to the wild type allele and the other three had sequence identical to the mutant allele identified by direct sequencing of the PCR product (data not shown).

PCR was then performed using DNA from four female members of the family and tailed exon 8 primers. The resulting products were gel purified and sequenced directly using BODIPY dye primer chemistry. Comparison of the resulting sequence to that obtained from the obligate carrier did not detect any mixed bases in any of these subjects, including IIB who had previously been identified as a carrier by skin biopsy (fig 1B).

To confirm the results of the sequencing experiments, PCR primers were designed which would specifically amplify either the mutant or the wild type allele (fig 2A). PCRs performed using either set of wild type specific primers successfully amplified a product of the expected size from all five family members. When the same PCR was performed using primer sets specific for the mutant allele, a product was only obtained using template DNA from the obligate carrier (fig 2B).

\section{Discussion}

Hunter syndrome shows a wide range of clinical phenotypes. In severely affected subjects the disease is characterised by progressive mental retardation, physical disability, and death occurring before the age of $15 .{ }^{17}$ The aim of this study was to perform carrier testing in a family affected by severe Hunter syndrome. The obligate carrier (IA) had two children who died of Hunter syndrome at the ages of $31 / 2$ and 11 years. Biochemical testing of skin biopsies from one of IA's daughters indicated that she was also a carrier of the disorder. These people are members of a more extensive pedigree consisting of at least nine affected subjects over five generations. Carrier detection in this case was complicated by the fact that no surviving affected subjects were available for study.

Direct dye primer sequencing of PCR products to identify mixed bases has previously been used within our laboratory to analyse the appearance of resistant strains of HIV in patients undergoing AZT treatment (Metzker et al, personal communication), and to detect polymorphisms within the CCR5 receptor. ${ }^{18}$ This study shows that the same approach can be used successfully to identify point mutations in DNA from carriers of Hunter syndrome. The approach itself uses standard PCR and 
fluorescent dye sequencing techniques and provides unambiguous carrier status information. A novel dye was used rather than the commercially available fluorescent dyes. This dye shows more uniform absorbence than the currently available commercial dyes and makes it possible to identify more accurately mixed bases within the resulting sequence (Metzker et al, personal communication).

The two point mutations identified in the obligate carrier resulted in both a missense and a nonsense mutation. Nonsense mutations have been observed to be associated with either an intermediate or severe phenotype, ${ }^{5}$ and it is assumed that the truncated proteins produced as a result of these mutations must have little or no enzymatic activity. To our knowledge, this is the first time either of these two mutations has been reported.

Following successful identification of the mutation within the obligate carrier, only the single exon containing the mutation need be amplified and sequenced from other family members. In addition to identifying the mutation in the obligate carrier accurately, we were able to use this approach to show that none of this subject's female descendants had inherited the mutant allele. This included one person who had previously been falsely identified as a carrier by biochemical testing.

An added advantage of this approach is that it will be equally affective when applied to families affected by the mild form of this disorder. In contrast, enzymatic testing requires careful attention to enzyme kinetics and can be unreliable when activity is in the normal range as there is overlap between the ranges of normal and heterozygous subjects. Biochemical testing also often requires analysis of multiple samples from the same person ${ }^{19}$ or testing by more than one approach ${ }^{620}$ to give a reliable result.

We thank Xiao-Mei Liu, Linda Savage, and Hua Shen for expert technical assistance. This work was supported in part by grants R01 HG00823 and P30 HG00210 from the National Human Genome Research Institute.

1 Wilson PJ, Morris CP, Anson DS, et al. Hunter syndrome: isolation of an iduronate-2-sulfatase cDNA clone and analysis of patient DNA. Proc Natl Acad Sci USA 1990;87:8531-5.

2 Flomen RH, Green EP, Green PM, Bentley DR, Giannelli F. Determination of the organization of coding sequences within the iduronate sulphate sulphatase (IDS) gene. Hum Mol Genet 1993;2:5-10

3 Timms KM, Lu F, Shen Y, et al. $130 \mathrm{~kb}$ of DNA sequence reveals two new genes and a regional duplication distal to the human iduronate-2-sulfate sulfatase locus. Genome Res 1995;5:71-8

4 Hopwood J, Bunge S, Morris CP, et al. Molecular basis of mucopolysaccharidosis type II: mutations in the iduronate2-sulphatase gene. Hum Mutat 1993;2:435-42.

5 Rathmann M, Bunge S, Beck M, Kresse H, TylkiSzymanska A, Gal A. Mucopolysaccharidosis type II (Hunter syndrome): mutation "hot spots" in the iduronate2-sulfatase gene. Am 7 Hum Genet 1996;59:1202-9.

6 Archer IM, Harper PS, Wusteman FS. An improved assay for iduronate-2-sulphate sulphatase in serum and its use in the detection of carriers of the Hunter syndrome. Clin Chim Acta 1981;112:107-12.

7 Yutaka T, Fluharty AL, Stevens RL, Kihara H. Iduronate sulfatase analysis of hair roots for identification of Hunter syndrome heterozygotes. Am f Hum Genet 1978;30:57582.

8 Nwokoro N, Neufeld EF. Detection of Hunter heterozygotes by enzymatic analysis of hair roots. Am $\mathcal{F}$ Hum Genet 1979;31:42-9.

9 Danes BS, Bearn AG. Hunter's syndrome: a genetic study of clones in cell culture with particular reference to the Lyon hypothesis. F Exp Med 1967;126:509-23.

10 Capobianchi MR, Romeo G. Mosaicism for sulfoiduronate sulfatase deficiency in carriers of Hunter's syndrome. Experimentia 1976;32:459-60.

11 Migeon BR, Sprenkle JA, Liebaers I, Scott JF, Neufeld EF $\mathrm{X}$-linked Hunter syndrome: the heterozygous phenotype in cell culture. Am F Hum Genet 1977;29:448-54.

12 Tønnesen T, Lykkelund C, Güttler F. Diagnosis of Hunter's syndrome carriers: radioactive sulphate incorporation into fibroblasts in the presence of fructose 1-phosphate. Hum Genet 1982;60:167-71.

13 Tennesen $T$. The use of fructose 1-phosphate to detect Hunter heterozygotes in fibroblast cultures from high-risk carriers. Hum Genet 1984;66:212-16.

14 Sambrook J, Fritsch EF, Maniatis T. Molecular cloning: a laboratory manual. 2nd ed. Cold Spring Harbor, NY: Cold Spring Harbor Laboratory Press, 1989.

15 Timms KM, Huckett LE, Belmont JW, Shapira SK, Gibbs RA. A DNA deletion confined to the iduronate-2-sulfatase promoter abolishes IDS gene expression. Hum Mutat 1998;11:121-6.

16 Metzker ML, Lu J, Gibbs RA. Electrophoretically uniform fluorescent dyes for automated DNA sequencing. Science 1996;271:1420-2.

17 Online Mendelian Inheritance in Man, OMIM ${ }^{\mathrm{TM}}$. Baltimore: Johns Hopkins University. MIM Number: 309900: 1/31/96. World Wide Web URL:http:// www3.ncbi.nlm.nih.gov/omim/.

18 Ansari-Lari MA, Liu XM, Metzker ML, Rut AR, Gibbs RA The extent of genetic variation in the CCR5 gene. Nat Genet 1997;16:221-2

19 Tønnesen T, Güttler F, Lykkelund C. Reliability of the use of fructose 1-phosphate to detect Hunter cells in fibroblast-cultures of obligate carriers of the Hunter syndrome. Hum Genet 1983;64:371-5.

20 Zlotogora J, Bach G. Heterozygote detection in Hunter syndrome. Am $\mathcal{F}$ Med Genet 1984;17:661-5. 